\title{
SCHRÖDINGER OPERATORS WITH A COMPLEX VALUED POTENTIAL
}

\author{
SOL SCHWARTZMAN \\ (Communicated by Chuu-Lian Terng)
}

\begin{abstract}
If $M_{n}$ is a compact Riemannian manifold for which $H^{1}\left(M_{n}, \mathbb{Z}\right)=$ $0, V$ is a continuous complex valued function whose imaginary part is of constant sign, and $-\Delta \psi+V \psi=0$ for some $C^{2}$ complex valued function $\psi$ on $M_{n}$, then either $\psi$ vanishes somewhere or there is a constant $c$ and an everywhere positive function $F$ such that $\psi=c F$.
\end{abstract}

Let $M_{n}$ be a smooth compact oriented connected Riemannian manifold for which $H^{1}\left(M_{n}, \mathbb{Z}\right)=0$. Let $\Lambda$ be the $n$-form on $M_{n}$ giving rise to the standard measure there, and let $V$ be a continuous complex valued function on $M_{n}$ such that either the imaginary part of $V$ is greater than or equal to zero everywhere or the imaginary part is less than or equal to zero everywhere.

Theorem 1. If $\psi$ is a complex valued function of class $C^{2}$ on $M_{n}$ such that $-\Delta \psi+$ $V \psi=0$, then either $\psi(x)=0$ for some $x$ or there is a constant $c$ and an everywhere positive function $F$ such that $\psi=c F$.

Proof. Suppose $\psi$ is different from 0 everywhere. Since $H^{1}\left(M_{n}, \mathbb{Z}\right)=0$, it follows that there exist real valued continuous functions $k$ and $u$ such that $\psi=e^{(k+i u)}$. Because $M_{n}$ is compact, there is at least one point $p$ at which $u$ assumes a maximum value.

It is known that one can always find a coordinate patch about $p$ in which $\Lambda=$ $d x_{1} \wedge \cdots \wedge d x_{n}$. In [2] it is proven that if $D_{\nabla u}$ denotes Lie differentiation with respect to the vector field $\nabla u$, then the imaginary part of $(\Delta \psi / \psi) e^{2 k} \Lambda$ equals $D_{\nabla u}\left(e^{2 k} \Lambda\right)$. A computation in [2] shows that in our special coordinate system,

$$
D_{\nabla u}\left(e^{2 k} \Lambda\right)=\left(\left[2 g^{i j} \frac{\partial k}{\partial x_{i}}+\frac{\partial g_{i j}}{\partial x_{i}}\right] \frac{\partial u}{\partial x_{j}}+g^{i j} \frac{\partial^{2} u}{\partial x_{i} \partial x_{j}}\right) e^{2 k} d x_{1} \wedge \cdots \wedge d x_{n} .
$$

Since $\Delta \psi / \psi=V$, if $\operatorname{Im}(V) \geq 0$ everywhere, then the same thing holds for the above expression. However, a theorem of E. Hopf [1] tells us that when $u$ satisfies an inequality of the form

$$
h^{j} \frac{\partial u}{\partial x_{j}}+g^{i j} \frac{\partial^{2} u}{\partial x_{i} \partial x_{j}} \geq 0
$$

in a connected region and assumes a maximum at some point in the region, then $u$ must be constant throughout the region.

Received by the editors May 19, 2011.

2010 Mathematics Subject Classification. Primary 35J10.

(C)2012 American Mathematical Society Reverts to public domain 28 years from publication 
The function $u$ that satisfies $\psi=e^{k+i u}$ must therefore be constant because the set of points at which $u$ assumes its maximum must be both open and closed and $M_{n}$ is connected. Hence $\psi$ equals a constant times a real valued function. Since $\psi$ vanishes nowhere, this real valued function must be of constant sign. It follows that $\psi$ is a constant times a positive function.

There is a parallel statement when

$$
h^{i} \frac{\partial u}{\partial x_{i}}+g^{i j} \frac{\partial^{2} u}{\partial x_{i} \partial x_{j}} \leq 0
$$

everywhere, so a similar argument shows that this assumption yields the same conclusion in this case.

If one assumes $V$ is real valued, then our present result yields the theorem at the end of [2] in the case where the manifold has no boundary.

Actually, the case where there is a boundary is more interesting than our present theorem, even though it only applies when $V$ is real valued, because the case where there is a boundary includes the situation in which $M_{n}$ is the closure of the interior of an $(n-1)$-sphere that is smoothly imbedded in $\mathbb{R}^{n}$.

It should be mentioned that the principal part of [2] is devoted to proving a theorem of the present type in the case in which $M_{n}$ is complete rather than compact. To compensate for the absence of compactness, one assumes that our real valued potential $V$ is bounded below.

\section{REFERENCES}

[1] Bochner, S. and Yano, K. Curvature and Betti Numbers, Princeton University Press, 1953 (p. 26). MR0062505(15:989f)

[2] Sol Schwartzman, Schrödinger Operators and the Zeroes of Their Eigenfunctions, Communications in Mathematical Physics 306 (2011), 187-191. MR2819423(2012e:81079)

Department of Mathematics, University of Rhode Island, Kingston, Rhode Island 02881

E-mail address: solschwartzman@gmail.com 